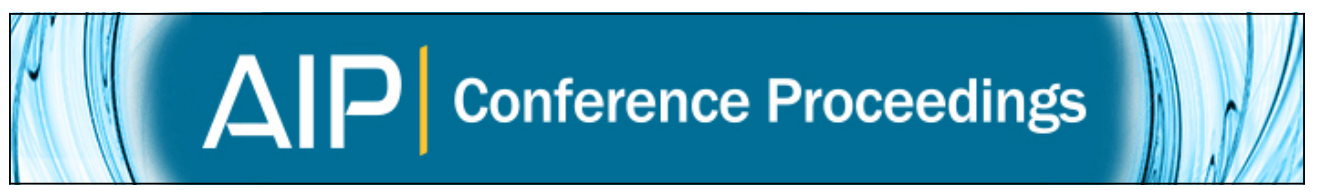

\title{
High-z X-ray Obscured Quasars in Galaxies with Extreme Mid-IR/Optical Colors
}

E. Piconcelli, G. Lanzuisi, F. Fiore, C. Feruglio, C. Vignali, M. Salvato, and C. Grappioni

Citation: AIP Conference Proceedings 1126, 231 (2009); doi: 10.1063/1.3149421

View online: http://dx.doi.org/10.1063/1.3149421

View Table of Contents:

http://scitation.aip.org/content/aip/proceeding/aipcp/1126?ver=pdfcov

Published by the AIP Publishing

\section{Articles you may be interested in}

Type 2 Quasars at the heart of dust-obscured galaxies (DOGs) at high z

AIP Conf. Proc. 1248, 493 (2010); 10.1063/1.3475316

X-ray obscured AGN in the GOODS-N

AIP Conf. Proc. 1248, 435 (2010); 10.1063/1.3475286

The IR to X-rays SED of the Heavily Obscured Quasar IRAS 09104+4109

AIP Conf. Proc. 1126, 242 (2009); 10.1063/1.3149424

The Extreme SED of the Quasar [HB89] 0102-272

AIP Conf. Proc. 1126, 235 (2009); 10.1063/1.3149422

The Infrared View of Luminous X-ray Selected Type 2 Quasars, and Coeval Nuclear Activity and Star Formation at $z=2$

AIP Conf. Proc. 1126, 227 (2009); 10.1063/1.3149420 


\title{
High-z X-ray Obscured Quasars in Galaxies with Extreme Mid-IR/Optical Colors
}

\author{
E. Piconcelli*, G. Lanzuisi ${ }^{\dagger}$, F. Fiore*, C. Feruglio**, C. Vignali ${ }^{\ddagger}$, , \\ M. Salvato and C. Gruppioni ${ }^{\S}$ \\ ${ }^{*}$ Osservatorio Astronomico di Roma, Via Frascati 33, I-00040 Monteporzio Catone, Italy \\ 'Dipartimento di Fisica, Università di Roma 'La Sapienza', P.le A. Moro 2, I-00185 Roma, Italy \\ ${ }^{* *}$ CEA, Irfu, Service d'Astrophysique, Centre de Saclay, 91191 Gif-sur-Yvette, France \\ ${ }^{\star}$ Dipartimento di Astronomia, Università di Bologna, Via Ranzani 1, I-40127 Bologna, Italy \\ \$Osservatorio Astronomico di Bologna (INAF), Via Ranzani 1, I-40127 Bologna, Italy \\ ${ }^{\top}$ Caltech, MC 105-24, 1200 East California Boulevard, Pasadena, CA 91125, USA
}

\begin{abstract}
Extreme Optical/Mid-IR color cuts have been used to uncover a population of dustenshrouded, mid-IR luminous galaxies at high redshifts. Several lines of evidence point towards the presence of an heavily absorbed, possibly Compton-thick quasar at the heart of these systems. Nonetheless, the X-ray spectral properties of these intriguing sources still remain largely unexplored. Here we present an X-ray spectroscopic study of a large sample of 44 extreme dust-obscured galaxies (EDOGs) with $F_{24 \mu \mathrm{m}} / F_{\mathrm{R}}>2000$ and $F_{24 \mu \mathrm{m}}>1.3 \mathrm{mJy}$ selected from a $6 \mathrm{deg}^{2}$ region in the SWIRE fields. The application of our selection criteria to a wide area survey has been capable of unveiling a population of $\mathrm{X}$-ray luminous, absorbed $\mathrm{z}>1$ quasars which is mostly missed in the traditional optical/X-ray surveys performed so far. Advances in the understanding of the X-ray properties of these recently-discovered sources by Simbol-Xobservations will be also discussed.
\end{abstract}

Keywords: Galaxies:active - Galaxies:nuclei - Infrared:galaxies - X-ray:galaxies PACS: $98.54 . \mathrm{Cm}-98.54 . \mathrm{Ep}$

\section{DUST OBSCURED QUASARS AT $z>1$}

A major obstacle in completing the census of accreting SMBH through different cosmic epoch arises in the detection of the heavily obscured AGN activity beyond the local Universe. In particular, the luminous end of the obscured AGN population (the region of type 2 quasars, QSO2 $\mathrm{s}^{1}$ hereafter) is poorly explored due to the interplay of two causes: (i) the fraction of obscured AGNs decreases with increasing luminosity [6]; (ii) the luminous QSO2s have low surface density and, therefore, large samples can be collected only by wide-area, multiwavelength (i.e. time-consuming) surveys.

In the recent years many works have used the mid-IR (MIR) emission or combinations of MIR and optical/radio data to successfully select a population of highly obscured AGNs at $z \gtrsim 1$ [3]. In particular, [12] found that most of dust obscured galaxies (DOGs hereafter) with $F_{24 \mu \mathrm{m}} / F_{\mathrm{R}} \gtrsim 1000$ and $24 \mu \mathrm{m}$ flux density $F_{24 \mu \mathrm{m}}>0.5 \mathrm{mJy}$ harbor a luminous, heavily obscured quasar at high $z$. Simultaneous starburst and obscured, powerful AGN activity has been observed in several z $>3$ DOGs with $F_{24 \mu \mathrm{m}} \gg 1 \mathrm{mJy}$,

\footnotetext{
${ }^{1}$ Here we define a type 2 QSO as an AGN with $L_{2-10}>10^{44} \mathrm{erg} \mathrm{s}^{-1}$ and $\mathrm{N}_{\mathrm{H}}>10^{22} \mathrm{~cm}^{-2}$, regardless the presence of broad lines in the optical spectrum.

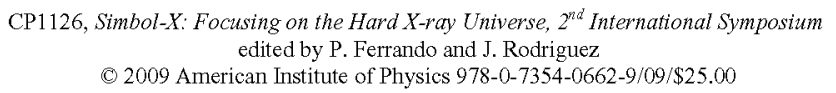


suggesting that these sources may represent systems caught in the process of host galaxy formation and intense SMBH growth [13]. This evolutionary phase, predicted by many theoretical models of galaxy evolution and formation [14], is rarely observed.

However, very few X-ray spectra of such intriguing X-ray faint, high-z DOGs have been investigated so far. We have therefore undertaken an exploratory program in order to study the largely unknown X-ray properties of the most extreme DOGs. X-ray data are indeed crucial in order to estimate their luminosity and the obscuring column of gas towards the nuclei of these objects, and to establish the fraction of QSO2s among them.

We refer the reader to i.e. [8] for the complete description of the selected sample and the detailed discussion of the results of this study.

\section{SELECTION CRITERIA AND RESULTS}

Our selection criterion consists in two conditions: (i) a $M I R / O \geq 2000$ and (ii) a $24 \mu \mathrm{m}$ flux density $F_{24 \mu \mathrm{m}} \geq 1.3 \mathrm{mJy}$. We applied these cuts to the MIR sources falling in five fields from the wide-area SWIRE survey. We refer to these sources as Extreme Dust Obscured Galaxies (EDOGs). We then retained only those EDOGs falling in a region covered by X-ray observations available in the $X M M-N e w t o n$ and Chandra archives. The $F_{24 \mu \mathrm{m}} / F_{\mathrm{R}}$ and $F_{24 \mu \mathrm{m}}$ cuts resemble those successfully employed by [2] and [12] to collect luminous, dust-enshrouded quasars at $z>1$ and by [4] to recover a large population of deeply buried, likely Compton-thick (CT) AGNs, being so X-ray faint to be largely undetected even in the $1 \mathrm{Ms}$ observation of the CDFS.

The final sample includes 44 EDOGs. The vast majority of spectroscopic (available for $16 \%$ of the sources) and photometric redshifts are in the range $0.7 \lesssim z \lesssim 2.5$, with an average $\langle z\rangle \simeq 1.2$.

When plotted in the MIR color-color diagnostic diagram proposed by [7], all but one (i.e. the source showing both the lowest redshift and the lowest MIR luminosity) EDOGS fall in the region typical of AGNs. Furthermore, most of them $(\sim 75 \%)$ are characterized by extremely red MIR colors, i.e. $\log \left(\mathrm{F}_{5.8} / \mathrm{F}_{3.6}\right) \gtrsim 0.4$ and $\log \left(\mathrm{F}_{8.0} / \mathrm{F}_{4.5}\right) \geq 0.4$, similarly to the highly luminous $\left(L_{5.8 \mu m}>10^{12} L_{\odot}\right)$ and obscured quasars at $z=1.3-3$ discovered by [12]. Figure 1a clearly shows how our simple selection if applied to a wide area $\left(6 \mathrm{deg}^{2}\right)$ allows us to collect very luminous objects. Our EDOGs are indeed, on average, $\gtrsim 1.5-2(\gtrsim 0.5)$ orders of magnitude brighter than the $F_{24 \mu \mathrm{m}} / F_{\mathrm{R}}>2000$ sources selected in the $<0.1(2) \mathrm{deg}^{2}$ CDFS(COSMOS) survey by $[4]([5])$.

Due to the inhomogeneous X-ray coverage, we were able to infer the X-ray spectral properties for only 23 out of 44 sources (hereafter referred as the $X$-ray sample). The major results from our analysis are the following: (i) almost all EDOGs exhibit evidence of X-ray obscuration $\left(\mathrm{N}_{\mathrm{H}} \geq 10^{22} \mathrm{~cm}^{-2}\right.$ ) (ii) all EDOGs in the $X$-ray sample show a 2$10 \mathrm{keV}$ luminosity $L_{2-10} \gg 10^{42} \mathrm{erg} \mathrm{s}^{-1}$, i.e. typical of AGN; (iii) $55 \%$ of the sources can be classified as QSO2 according to their $\mathrm{N}_{\mathrm{H}}$ and $L_{2-10}$; and (iv) CT QSO2s at $z>1.4$ account for $13 \%$ of the sample. A summary of the X-ray spectral properties of our EDOGs can be found in Table 1. We also report the results obtained for a control sample of 20 sources showing hard X-ray flux $\left(F_{2-10} \approx 10^{-15}-10^{-13} \mathrm{erg} \mathrm{cm}^{-2} \mathrm{~s}^{-1}\right)$ and redshift distribution similar to those of the $X$-ray sample, collected from a blind search 

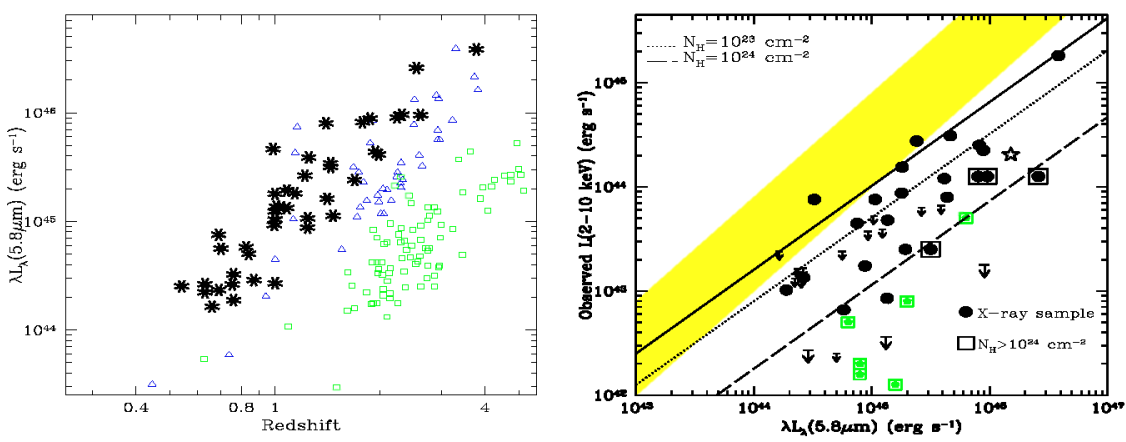

FIGURE 1. Left (a): the $L_{5.8 \mu m}-z$ plane for COSMOS (open triangles) and CDFS sources (open squares) with $F_{24 \mu \mathrm{m}} / F_{\mathrm{R}}>2000$. Our EDOGs are indicated by asterisks. Right (b): $L_{5.8 \mu m}$ vs observed $L_{2-10}^{O b_{S}}$. Downward-pointing arrows indicate the $3 \sigma$ upper limit on $L_{2-10}^{O b s}$ for the X-ray undetected sources. The solid line represents the relationship between $L_{2-10}$ and $L_{5.8 \mu m}$ obtained for the $\mathrm{X}$-ray sample, while the dotted(dashed) line represents the $L_{2-10} / L_{5.8 \mu m}$ ratio expected for an absorption with $\mathrm{N}_{\mathrm{H}}=10^{23}\left(10^{24}\right)$ $\mathrm{cm}^{-2}$. The shaded area indicates the range of $L_{2-10}-L_{5.8 \mu \mathrm{m}}$ found for AGNs in the local Universe [10]. Small pentagons are the CT AGNs from [1] and the empty star indicates the powerful, dust-enshrouded QSO2 IRAS 09104 +4109 at $z=0.442$ (e.g. [11] and [9])

TABLE 1. The source breakdown according to the absorption properties. ${ }^{a}$ we report the maximum (minimum) value derived assuming the best fit value (the lowest value at the $90 \%$ confidence level) of the $\mathrm{N}_{\mathrm{H}}$.

\begin{tabular}{lcccc}
\hline Sample & $\mathbf{N}_{\mathrm{H}}<10^{22} \mathbf{c m}^{-2}$ & $\mathbf{N}_{\mathrm{H}} \geq 10^{22} \mathbf{c m}^{-2}$ & QSO2s & No. of sources \\
\hline$X$-ray & $13-52 \%$ & $48-87 \%$ & $43-60 \%$ & 23 \\
Control & $85 \%$ & $15 \%$ & $5 \%$ & 20 \\
\hline
\end{tabular}

in the same $6 \mathrm{deg}^{2}$ SWIRE area considered for our selection.

\section{DISCUSSION AND CONCLUSIONS}

A remarkably high fraction of EDOGs in the $X$-ray sample can be classified as heavily absorbed QSO2s. This is a very important result, given the paucity of members of this class of elusive AGNs among sources that are typically detected in hard X-ray surveys [15]. The presence of a powerful, hidden quasar in these extreme $F_{24 \mu \mathrm{m}} / F_{\mathrm{R}}$ objects may represent a crucial phase in the formation and evolution of their host galaxies.

The solid line in Fig. $2 \mathrm{~b}$ represents the relationship between $L_{2-10}$ and $L_{5.8 \mu m}$ derived for the EDOGs in the X-ray sample. A clear shift from the trend measured for AGNs in the local Universe can be observed at the highest MIR luminosities. Larger $L_{5.8 \mu \mathrm{m}} / L_{2-10}$ ratios for the most luminous MIR objects suggest that an evolution of this ratio with $z$ is a possible explanation, since high $L_{5.8 \mu \mathrm{m}}$ sources are found at high $z$ owing to our selection criterion (e.g. Fig la). We derived a rough estimate of the $\mathrm{N}_{\mathrm{H}}$ value also for the $15 \mathrm{X}$-ray undetected EDOGs showing the reddest MIR colors which are typical 

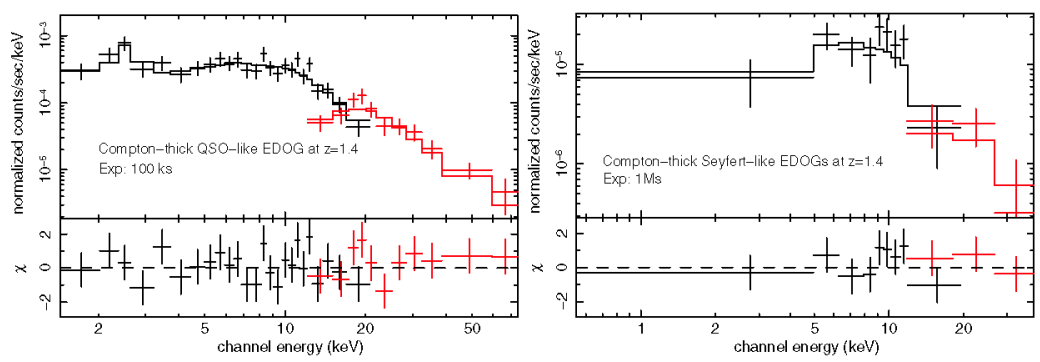

FIGURE 2. (a) Left: $100 \mathrm{ks}$ Simbol- $X$ SSD + CZT simulated spectrum of a CT quasar-like EDOG at $z=1.4\left(L_{2-10}=10^{45} \mathrm{erg} \mathrm{s}^{-1} ; F_{2-10}=2.5 \times 10^{-14} \mathrm{erg} \mathrm{cm}^{-2} \mathrm{~s}^{-1}\right)$. (b) Right: $1 \mathrm{Ms}$ SSD + CZT simulated spectrum of a CT Seyfert-like EDOG at $z=1.4\left(L_{2-10}=10^{43} \mathrm{erg} \mathrm{s}^{-1} ; F_{2-10}=8 \times 10^{-16} \mathrm{erg} \mathrm{cm}^{-2} \mathrm{~s}^{-1}\right)$

of MIR AGN-dominated objects ([12]), by comparing the intrinsic 2-10 keV luminosity expected on the basis of the value of $L_{5.8 \mu m}$ with the observed (i.e. not absorption corrected) $L_{2-10}^{O b s}$ estimated from the upper limit on the $2-10 \mathrm{keV}$ flux. We found that $\sim 30 \%$ of these X-ray faint EDOGs are consistent with being CT AGNs.

Heavily obscured X-ray sources as EDOGs are ideal targets for Simbol-X. Due to their extreme faintness and large redshift, the study of their X-ray properties will benefit of the high throughput and broad energy coverage offered by Simbol- $X$. Therefore, they will surely represent a very important scientific topic for this future X-ray mission. We present two simulations that well illustrate the unprecedented spectroscopic quality that can be achieved by realistic Simbol-X observations of EDOGs. Fig. 2a shows the simulated SSD +CZT spectrum obtained in a $100 \mathrm{ks}$ exposure for a $E D O G$ at $z=1.4$ containing a CT QSO2 (i.e. $L_{2-10}=10^{45} \mathrm{erg} \mathrm{s}^{-1}$ ) with a $F_{2-10}=2.5 \times 10^{-14} \mathrm{erg} \mathrm{cm}^{-2}$ $\mathrm{s}^{-1}$, i.e. the characteristic value measured for our sample. Furthermore, a $1 \mathrm{Ms}$ Simbol$X$ exposure (i.e. a typical deep field) will enable for the first time the exploration of the spectral properties of a CT Seyfert-like (i.e. $L_{2-10}=10^{43} \mathrm{erg} \mathrm{s}^{-1}$ ) $E D O G$ at $z=1.4$ with an extremely faint $F_{2-10}=8 \times 10^{-16} \mathrm{erg} \mathrm{cm}^{-2} \mathrm{~s}^{-1}$ (e.g. Fig. 2b).

\section{REFERENCES}

1. Alexander, D. M., et al. 2008, ApJ, 687, 835

2. Dey, A., et al. $2008, \mathrm{ApJ}, 677,943$

3. Donley et al. 2008, ApJ, 687, 111

4. Fiore, F., et al. 2008, ApJ, 672, 94

5. Fiore, F., et al. 2008b, arXiv:0810.0720

6. Hasinger, G. 2008, arXiv:0808.0260

7. Lacy, M., Storrie-Lombardi, L. J., Sajina, A., et al. 2004, ApJS, 154, 166

8. Lanzuisi, G., Piconcelli, E., Fiore, F., et al. 2009a, A\&A, arXiv:0902.2517

9. Lanzuisi, G., Piconcelli, E., Fiore, F., et al. 2009, this Volume

10. Lutz, D., et al. 2004, A\&A, 418, 465

11. Piconcelli, E., et al. 2007, A\&A, 473,85

12. Polletta, M., et al. 2008a, ApJ, 675,960

13. Polletta, M., et al. 2008b, A\&A, 492,81

14. Silk, J., \& Rees, M. J. 1998, A\&A, 331, L1

15. Tozzi, P., Gilli, R., Mainieri, V., et al., 2006, A\&A, 451, 457 